Ann. Funct. Anal. 6 (2015), no. 4, 134-154

http://doi.org/10.15352/afa/06-4-134

ISSN: 2008-8752 (electronic)

http://projecteuclid.org/afa

\title{
MAXIMAL BILINEAR CALDERÓN-ZYGMUND OPERATORS OF TYPE $\omega(t)$ ON NON-HOMOGENEOUS SPACE
}

\author{
TAOTAO ZHENG ${ }^{1,2 *}$, ZHENG WANG ${ }^{2}$ AND WEILIANG XIAO ${ }^{2}$ \\ Communicated by K. F. Taylor
}

\begin{abstract}
Let $(\mathcal{X}, d, \mu)$ be a geometrically doubling metric space and assume that the measure $\mu$ satisfies the upper doubling condition. In this paper, the authors, by invoking a Cotlar type inequality, show that the maximal bilinear Calderón-Zygmund operators of type $\omega(t)$ is bounded from $L^{p_{1}}(\mu) \times L^{p_{2}}(\mu)$ into $L^{p}(\mu)$ for any $p_{i} \in(1, \infty]$ and bounded from $L^{p_{1}}(\mu) \times L^{p_{2}}(\mu)$ into $L^{p, \infty}(\mu)$ for $p_{1}=1$ or $p_{2}=1$, where $p \in[1 / 2, \infty), 1 / p_{1}+1 / p_{2}=1 / p$. Moreover, if $\vec{w}=\left(w_{1}, w_{2}\right)$ belongs to the weight class $A_{\vec{p}}^{\rho}(\mu)$, using the John-strömberg maximal operator and the John-strömberg sharp maximal operator, the authors obtain a weighted weak type estimate $L^{p_{1}}\left(w_{1}\right) \times L^{p_{2}}\left(w_{2}\right) \rightarrow L^{p, \infty}\left(v_{\vec{w}}\right)$ for the maximal bilinear Calderón-Zygmund operators of type $\omega(t)$. By weakening the assumption of $\omega \in \operatorname{Dini}(1 / 2)$ into $\omega \in \operatorname{Dini}(1)$, the results obtained in this paper are substantial improvements and extensions of some known results, even on Euclidean spaces $\mathbb{R}^{n}$.
\end{abstract}

\section{INTRODUCTION AND MAIN RESULTS}

As we all know, the Calderón-Zygmund theory has played an important role in harmonic analysis. We may note that the underlying measure of these works possess the measure doubling property,

$$
\mu(B(x, 2 r)) \leq C \mu(B(x, r)),
$$

where $\mu$ is a Borel measure, the ball $B$ denotes $B(x, r)=\{y \in \mathcal{X}: d(x, y)<r\}$, which is equipped with a fixed center $x \in \mathcal{X}$ and radius $r>0$. A metric space $(\mathcal{X}, d)$ equipped with such a measure $\mu$ is called a space of homogeneous type.

Date: Received: Dec. 17, 2014; Accepted: Mar. 25, 2015.

* Corresponding author.

2010 Mathematics Subject Classification. Primary 42B25; Secondary 46E30, 42B20.

Key words and phrases. Bilinear Calderón-Zygmund operator, sharp maximal operator, nonhomogeneous space, upper doubling measures, weight. 
However, recently, considerable attention has been paid to the study of when the underlying measure only satisfies the polynomial growth condition, namely, there exist positive $C_{0}$ and $n$ such that, for all $x \in \mathcal{X}$ and $r \in(0, \infty)$,

$$
\mu(B(x, r)) \leq C_{0} r^{n}
$$

Although such a measure does not satisfy the doubling condition, many results on the classical Calderón-Zygmund theory have been proved to still hold; see, for example, $[8,16,17]$ and some references therein.

We may also notice that the underlying measure satisfying the polynomial growth condition (1.2) are different from, not more general than satisfying (1.1). Recently, Hytönen [9] gave a new class of metric measure spaces $(\mathcal{X}, d, \mu)$, which are called non-homogeneous spaces. The new class of metric measure spaces are sufficiently general to include in a natural way both the space of homogeneous type and a metric space with polynomial growth condition, where the measure $\mu$ satisfies the upper doubling condition (see Definition 1.2). The following notions of geometrically doubling and upper doubling measures $\mu$ are originally from Hytönen [9].

Definition 1.1. A metric space $(\mathcal{X}, d)$ is called geometrically doubling if there exists a number $N \in \mathbb{N}$ such that any open ball $B(x, r) \subset \mathcal{X}$ can be covered by at most $N$ balls $B\left(x_{i}, \frac{r}{2}\right)$.

Definition 1.2. A Borel measure $\mu$ in the metric space $(\mathcal{X}, d, \mu)$ is said to be an upper doubling measure if there exists a dominating function $\lambda: \mathcal{X} \times \mathbb{R}_{+} \rightarrow \mathbb{R}_{+}$ and a constant $C_{\lambda}$ such that:

(1) For any fixed $x \in \mathcal{X}, r \longmapsto \lambda(x, r)$ is increasing.

(2) $\lambda(x, 2 r) \leq C_{\lambda} \lambda(x, r)$.

(3) The inequality $\mu(B(x, r)) \leq \lambda(x, r) \leq C_{\lambda} \lambda(x, r / 2)$ holds for all $x \in \mathcal{X}, 0<$ $r<\infty$.

(4) $\lambda(x, r) \approx \lambda(y, r)$ for all $r>0, x, y \in \mathcal{X}$ and $d(x, y) \leq r$.

When $\lambda(x, r)=\mu(B(x, r))$, the measure doubling is a special case of upper doubling. If we take $\lambda(x, r)$ equal to $C_{0} r^{n}$, the measure $\mu$ as in (1.2) on $\mathbb{R}^{n}$ is also an upper doubling measure.

In this paper, we assume that $(\mathcal{X}, d, \mu)$ is a geometrically doubling metric space and the measure $\mu$ is an upper doubling measure.

Bui and Doung [2] established a Calderón-Zygmund decomposition on $(\mathcal{X}, d, \mu)$ and obtained some properties about the boundedness of Calderón-Zygmund operator on various function spaces. In the form of a Cotlar inequality, they also obtain the boundedness of maximal Calderón-Zygmund operator.

We also note that multilinear Calderón-Zygmund theory has been studied by many researchers, we can see [3, 5, 7, 11, 13]. Grafakos and Torres [6] investigated the boundedness of maximal multilinear Calderón-Zygmund operator on product of Lebesgue spaces. Moreover, some weighted norm inequalities are also obtained for this maximal operator. Recently, Maldonado and Naibo [13] developed a theory of the bilinear Calderón-Zygmund operator of type $\omega(t)$ on Euclidean $\mathbb{R}^{n}$ and applied them to the investigation of para-products and bilinear pseudodifferential operators with mild regularity. They obtained some properties of 
the maximal bilinear Calderón-Zygmund operator of type $\omega(t)$, as well as some weighted estimates, where $\omega \in \operatorname{Dini}(1 / 2)$ (see (1.3)).

However, there are few topics about multilinear singular integral on $(\mathcal{X}, d, \mu)$. $\mathrm{Hu}$, Meng and Yang [7] established some weighted norm inequalities for the multilinear Calderón-Zygmund operators on the non-homogeneous spaces. Zheng et al.[19] extended the bilinear Calderón-Zygmund operator of type $\omega(t)$ on the non-homogeneous spaces and weakened the assumption of $\omega \in \operatorname{Dini}(1 / 2)$ to $\omega \in \operatorname{Dini}(1)$.

Inspired by [7, 13, 19], it is natural to raise the following question:

How to establish corresponding results about maximal bilinear Calderón-Zygmund operator of type $\omega(t)$ on non-homogeneous spaces $(\mathcal{X}, d, \mu)$ ?

The question is not motivated only by a mere quest to extend the bilinear Calderón-Zygmund operators of type $\boldsymbol{\omega}(t)$ from the classical Calderón-Zygmund theory, but rather by their natural appearance in analysis (see [5, 13, 18]).

To state the main results, we now give the definition of bilinear CalderónZygmund operator of type $\omega(t)$ and the corresponding maximal bilinear operators of type $\omega(t)$ on $(\mathcal{X}, d, \mu)$.

For $a>0$, we write $\omega \in \operatorname{Dini}(a)$ if $\omega:[0, \infty) \rightarrow[0, \infty), \omega$ is nondecreasing and

$$
|\omega|_{\operatorname{Dini}(a)}:=\int_{0}^{1} \omega^{a}(t) \frac{d t}{t}<\infty .
$$

It is obvious that

$$
\int_{0}^{1} \omega(t) \frac{d t}{t}=\int_{0}^{1} \omega^{\frac{1}{2}}(t) \omega^{\frac{1}{2}}(t) \frac{d t}{t} \leq \omega^{\frac{1}{2}}(1) \int_{0}^{1} \omega^{\frac{1}{2}}(t) \frac{d t}{t} .
$$

Denote

$$
\frac{1}{\lambda(x, d(x, \tilde{y}))}=\min _{i \in\{1,2\}}\left\{\frac{1}{\lambda\left(x, d\left(x, y_{i}\right)\right)}\right\}
$$

Definition 1.3. Let $K\left(x, y_{1}, y_{2}\right)$ be a locally integrable function defined away from the diagonal $x=y_{1}=y_{2}$ in $(\mathcal{X})^{3}$ and $\omega:[0, \infty) \rightarrow[0, \infty)$ be a nondecreasing function. We say that $K\left(x, y_{1}, y_{2}\right)$ is a bilinear Calderón-Zygmund kernel of type $\omega(t)$ if it satisfies the following size estimate

$$
\left|K\left(x, y_{1}, y_{2}\right)\right| \leq \frac{A}{[\lambda(x, d(x, \tilde{y}))]^{2}}
$$

for some $A>0$ and $\left(x, y_{1}, y_{2}\right) \in(\mathcal{X})^{3}$ with $x \neq y_{i}$ for some $i$. Furthermore, we have the smoothness estimates

$$
\left|K\left(x, y_{1}, y_{2}\right)-K\left(x^{\prime}, y_{1}, y_{2}\right)\right| \leq \frac{A}{[\lambda(x, d(x, \tilde{y}))]^{2}} \omega\left(\frac{d\left(x, x^{\prime}\right)}{\sum_{i=1}^{2} d\left(x, y_{i}\right)}\right)
$$

whenever $d\left(x, x^{\prime}\right) \leq \frac{1}{2} \max _{i \in\{1,2\}} d\left(x, y_{i}\right)$ and also that

$$
\left|K\left(x, y_{1}, y_{2}\right)-K\left(x, y_{1}^{\prime}, y_{2}\right)\right| \leq \frac{A}{[\lambda(x, d(x, \tilde{y}))]^{2}} \boldsymbol{\omega}\left(\frac{d\left(y_{1}, y_{1}^{\prime}\right)}{\sum_{i=1}^{2} d\left(x, y_{i}\right)}\right)
$$




$$
\left|K\left(x, y_{1}, y_{2}\right)-K\left(x, y_{1}, y_{2}^{\prime}\right)\right| \leq \frac{A}{[\lambda(x, d(x, \tilde{y}))]^{2}} \omega\left(\frac{d\left(y_{2}, y_{2}^{\prime}\right)}{\sum_{i=1}^{2} d\left(x, y_{i}\right)}\right)
$$

whenever $d\left(y_{i}, y_{i}^{\prime}\right) \leq \frac{1}{2} \max _{i \in\{1,2\}} d\left(x, y_{i}\right)$.

A bilinear operator $T$ is said to be associated with a bilinear Calderón-Zygmund kernel of type $\omega(t)$, if for $f_{1}, f_{2}$ are $L^{\infty}$ function with compact support and $x \notin \cap_{i=1}^{2} \operatorname{supp} f_{i}$.

$$
T\left(f_{1}, f_{2}\right)(x)=\int_{(\mathcal{X})^{2}} K\left(x, y_{1}, y_{2}\right) f_{1}\left(y_{1}\right) f_{2}\left(y_{2}\right) d \mu\left(y_{1}\right) d \mu\left(y_{2}\right) .
$$

If the bilinear operator $T$ is associated with $K\left(x, y_{1}, y_{2}\right)$ and can be extended from $L^{r_{1}}(\mu) \times L^{r_{2}}(\mu)$ into $L^{r, \infty}(\mu)$ for some $1<r_{i}<\infty(i=1,2)$ and $r>1$ with $\sum_{i=1}^{2} \frac{1}{r_{i}}=\frac{1}{r}$, or from $L^{r_{1}}(\mu) \times L^{r_{2}}(\mu)$ into $L^{1}(\mu)$ for some $1<r_{i}<\infty(i=1,2)$ and $\sum_{i=1}^{2} \frac{1}{r_{i}}=1$, then $T$ is said to be a bilinear Calderón-Zygmund operator of type $\omega(t)$.

Throughout this paper, the bilinear operator $T$ associated kernel $K\left(x, y_{1}, y_{2}\right)$ is assumed to be that

$$
L^{1}(\mu) \times L^{1}(\mu) \rightarrow L^{1 / 2, \infty}(\mu)
$$

and to satisfy that for all bounded functions $f_{1}, f_{2}$ with bounded support and $\mu$-almost every $x \in \mathcal{X} \backslash\left(\bigcap_{j=1}^{2} \operatorname{supp}\left(f_{j}\right)\right)$. Let $W$ be the norm of $T$ in (1.9).

For $\varepsilon>0$, define the truncated operator $T_{\varepsilon}$ by setting, for all $x \in \mathcal{X}$,

$$
T_{\varepsilon}\left(f_{1}, f_{2}\right)(x)=\int_{\max \left\{d\left(x, y_{1}\right), d\left(x, y_{2}\right)\right\}>\varepsilon} K\left(x, y_{1}, y_{2}\right) f_{1}\left(y_{1}\right) f_{2}\left(y_{2}\right) d \mu\left(y_{1}\right) d \mu\left(y_{2}\right) .
$$

The maximal bilinear Calderón-Zygmund operator of type $\omega(t)$ is defined by setting, for all $x \in \mathcal{X}$,

$$
T^{\star}\left(f_{1}, f_{2}\right)(x)=\sup _{\varepsilon>0}\left|T_{\varepsilon}\left(f_{1}, f_{2}\right)(x)\right| .
$$

The classical Calderón-Zygmund operator of type $\omega(t)$ was studied by Yabuta [18]. Our first goal in this paper is to obtain the boundedness of the maximal bilinear Calderón-Zygmund operators of type $\omega(t)$ on the non-homogeneous spaces $(\mathcal{X}, d, \mu)$.

Theorem 1.4. Consider $\omega \in \operatorname{Dini}(1)$. Let $K$ be a $\mu$-locally integrable function defined away from the diagonal $x=y_{1}=y_{2}$ in $(\mathcal{X})^{3}$, which satisfies (1.4), (1.5), (1.6) and (1.7) . $T^{\star}$ is the maximal bilinear Calderón-Zygmund operators of type $\omega(t)$ defined as in (1.10). Assume $1 / p_{1}+1 / p_{2}=1 / p$, then

(i) $T^{\star}$ can be extended to a bounded operator from $L^{p_{1}}(\mu) \times L^{p_{2}}(\mu)$ into $L^{p}(\mu)$ for $1<p_{1}, p_{2} \leq \infty, 1 / 2 \leq p<\infty$.

(ii) $T^{\star}$ can also be extended to a bounded operator from $L^{p_{1}}(\mu) \times L^{p_{2}}(\mu)$ into $L^{p, \infty}(\mu)$ for $p_{1}=1$ or $p_{2}=1$.

Remark 1.5. Our conclusions improved the corresponding results in [13] by reducing the condition of $\omega \in \operatorname{Dini}(1 / 2)$ to $\omega \in \operatorname{Dini}(1)$, even when $(\mathcal{X}, d, \mu)=$ $\left(\mathbb{R}^{n},|\cdot|, d x\right)$. On the other hand, since the concavity of $\omega$ implies the doubling property of $\omega$, which is not needed in Theorem 1.4, we can remove the concavity. 
Definition 1.6. [7] Let $\rho \in[1, \infty), \vec{p}=\left(p_{1}, p_{2}\right)$ with $p_{1}, p_{2} \in[1, \infty)$ and $1 / p_{1}+$ $1 / p_{2}=1 / p$. A map $\vec{w}=\left(w_{1}, w_{2}\right)$ is said to belong to $A_{\vec{p}}^{\rho}(\mu)$ if $w_{1}$ and $w_{2}$ are negative $\mu$-measurable functions and there exists a positive constant $C$ such that for all balls $B \subset \mathcal{X}$,

$$
\left\{\frac{1}{\mu(\rho B)} \int_{B} v_{\vec{w}}(x) d \mu(x)\right\} \prod_{i=1}^{2}\left\{\frac{1}{\mu(\rho B)} \int_{B}\left[w_{i}(x)\right]^{1-p_{i}^{\prime}} d \mu(x)\right\}^{p / p_{i}^{\prime}} \leq C,
$$

where, for all $x \in \mathcal{X}, v_{\vec{w}}(x)=\prod_{i=1}^{2}\left[w_{i}(x)\right]^{p / p_{i}^{\prime}}$ and, when $p_{i}=1$,

$$
\left\{\frac{1}{\mu(\rho B)} \int_{B}\left[w_{i}(x)\right]^{1-p_{i}^{\prime}} d \mu(x)\right\}^{p / p_{i}^{\prime}}
$$

is understood as $\left(\inf _{B} w_{i}\right)^{-1}$ for $i \in\{1,2\}$. For $a>0$, the notation $a B:=$ $B\left(x, a r_{B}\right)$ stands for the concentric dilation of $B$, where the radius of $B$ are denoted by $r_{B}$.

When $\rho=1$ and $(\mathcal{X}, d, \mu)=\left(\mathbb{R}^{n},|\cdot|, d x\right)$, the weight class $A_{\vec{p}}^{\rho}(\mu)$ is introduced by Lerner et al. in [11].

The second goal is to obtain a weighted estimate for the maximal bilinear Calderón-Zygmund operator of type $\omega(t)$ on $(\mathcal{X}, d, \mu)$. As pointed out by Orobitg and Pérez [15], without the additional assumption that the faces of any ball have $\mu$ measure zero, it is still unclear whether the reverse Hölder's inequality holds for $u \in A_{\vec{p}}^{\rho}(\mu)$, so we can only obtain the weighted weak type estimate under the given conditions.

Theorem 1.7. Consider $\omega \in \operatorname{Dini}(1)$. Let $K\left(x, y_{1}, y_{2}\right)$ be a locally integrable function defined away from the diagonal $x=y_{1}=y_{2}$ in $(\mathcal{X})^{3}$, which satisfies (1.4), (1.5), (1.6) and (1.7). Let $T^{\star}$ be defined as in (1.10). Then for any $p_{1}, p_{2} \in[1, \infty)$ with $1 / p=1 / p_{1}+1 / p_{2}$ and $\vec{w}=\left(w_{1}, w_{2}\right) \in A_{\vec{p}}^{\rho}(\mu)$, there exists a constant $C$ such that for all $f_{1} \in L^{p_{1}}\left(w_{1}\right)$ and $f_{2} \in L^{p_{2}}\left(w_{2}\right)$,

$$
\left\|T^{\star}\left(f_{1}, f_{2}\right)\right\|_{L^{p, \infty}}\left(v_{\vec{w}}\right) \leq C\left\|f_{1}\right\|_{L^{p_{1}\left(w_{1}\right)}}\left\|f_{2}\right\|_{L^{p_{2}\left(w_{2}\right)}} .
$$

Remark 1.8. Lu and Zhang [12] established the end-point weak type estimate for the bilinear Calderón-Zygmund operator $T$ of type $\boldsymbol{\omega}(t)$ on Euclidean $\mathbb{R}^{n}$, as well as some weighted estimates. We may notice that in their results the underlying measure $\mu$ is the Lebesgue measure which satisfies (1.1). However, in our case $\mu$ satisfies only the upper doubling condition, the estimates may become even more complicated and many extra difficulties might raise, due to a weak structure of the space $(\mathcal{X}, d, \mu)$. For example, how to establish a bilinear Calderón-Zygmund decomposition on $(\mathcal{X}, d, \mu)$ and how to prove the end-point weak type estimate (1.9) for the bilinear Calderón-Zygmund operator. To our best knowledge, these problems have not been solved so far. We also want to point out that although we state our results on the bilinear case, all results are valid in the multilinear case without any essential difference and difficulty in the proof. With our results, one can establish some estimates on multilinear para product and multilinear pseudo-differential operators on the space $(\mathcal{X}, d, \mu)$ (see [12] for this easy fact). These works are related to results in $[1,4,10,14]$. 
Finally, we fix some notations and define some terminologies. For $\alpha, \beta>1$, a ball $B \subset \mathcal{X}$ is said $(\alpha, \beta)$-doubling if $\mu(\alpha B) \leq \beta \mu(B)$. Hytönen [9] pointed

out that for any $\alpha \in(1, \infty), \beta \in\left(C_{\lambda}^{\log _{2} \alpha}, \infty\right)$ and any ball $B \subset \mathcal{X}$, there there exists some $j \in \mathbb{Z}_{+}$such that $\alpha^{j} B$ is $(\alpha, \beta)$-doubling. In what follows, for a fixed $\rho \in[1, \infty)$, by a doubling ball $B$, we always mean that $B$ is a $\left(30 \rho, \beta_{30 \rho}\right)$-doubling ball with

$$
\beta_{30 \rho}>\max \left\{(30 \rho)^{3 n}, C_{\lambda}^{3 \log _{2}(30 \rho)}\right\} .
$$

For any $\rho \in[1, \infty)$ and ball $B \subset \mathcal{X}, \widetilde{B}$ denotes the smallest $\left(30 \rho, \beta_{30}\right)$-doubling ball of the form $(30 \rho)^{j} B$ with $j \in \mathbb{Z}_{+}$. For any two balls $B \subset S$, we define

$$
K_{B, S}=\int_{2 S \backslash B} \frac{1}{\lambda\left(x_{B}, d\left(x, x_{B}\right)\right)} d \mu(x) .
$$

For all balls $B \subset R \subset S$, we have $K_{B, R} \leq K_{B, S}$ and for $\rho \in[1, \infty)$, there exists a positive constant $C$, depending on $\rho$, such that for all balls $B \subset S$ with $r_{S}<\rho r_{B}$, $K_{B, S} \leq C$.

We denote $L^{p}(\mathcal{X}, \mu)$ by $L^{p}(\mu)$ for brevity. For $p \geq 1, p^{\prime}=p /(p-1)$ denotes the dual exponent of $p$. The letter $C$ always denotes a positive constant that may vary at each occurrence, but is independent of all essential variables. The symbol $f \lesssim g$ means that there exists a positive constant $C$ such that $f \leq C g$.

\section{Cotlar type inequality and proof of Theorem 1.4}

In this section, we will establish a lemma and a Cotlar type inequality. From these, it is easy to deduce the desired result in Theorem 1.4.

Lemma 2.1. Suppose $K$ is a $\mu$-locally integrable function, which satisfies (1.4), (1.5), (1.6) and (1.7). The bilinear operator $T$ is defined as in (1.8). Assume $\omega \in \operatorname{Dini}(1), 1 / 2 \leq p<\infty, 1 / p_{1}+1 / p_{2}=1 / p, f_{1} \in L^{p_{1}}(\mu)$ and $f_{2} \in L^{p_{2}}(\mu)$. Then $T$ extends boundedness from $L^{p_{1}}(\mu) \times L^{p_{2}}(\mu)$ into $L^{p}(\mu)$ for any $p_{1}, p_{2} \in$ $(1, \infty]$ and from $L^{p_{1}}(\mu) \times L^{p_{2}}(\mu)$ into $L^{p, \infty}(\mu)$ for $p_{1}=1$ or $p_{2}=1$.

Lemma 2.1 can be proved by modifying the Theorem 1.5 in [19], we omit the details of proof for brevity.

Next, we will introduce some maximal operator associated with the Cotlar type inequality, which plays an important role in the proof of Theorem 1.4.

Let $p \in(1, \infty), s \in(0, \infty)$ and $\tau \in(0, \infty)$. The following maximal operators are defined by setting for all $f \in L^{p}(\mu)$ and $x \in \mathcal{X}$,

$$
\begin{gathered}
M_{(\tau), s} f(x)=\sup _{B \ni x}\left\{\frac{1}{\mu(\tau B)} \int_{B}|f(y)|^{s} d \mu(y)\right\}^{\frac{1}{s}}, \\
M_{(\tau)} f(x)=\sup _{B \ni x} \frac{1}{\mu(\tau B)} \int_{B}|f(y)| d \mu(y) .
\end{gathered}
$$

If $s=1, M_{(\tau), s}$ is $M_{(\tau)}$. It was pointed out by Hytönen [9] that $M_{(\tau), s}$ and $M_{(\tau)}$ are bounded from $L^{p}(\mu)$ into itself with $p \in(1, \infty)$ and from $L^{1}(\mu)$ into $L^{1, \infty}(\mu)$ for $\tau \geq 5$. 
We also need the multilinear maximal operator $\mathcal{M}_{(\eta)}(\eta>1)$,

$$
\mathcal{M}_{(\eta)}\left(f_{1}, f_{2}\right)(x)=\sup _{B \ni x} \prod_{i=1}^{2} \frac{1}{\mu(\eta B)} \int_{B}\left|f_{i}\left(y_{i}\right)\right| d \mu\left(y_{i}\right) .
$$

which is introduced by Lerner [11] when $\mu$ is Lebesgue measure and $\eta=1$. It is obvious that the operator $\mathcal{M}_{(\eta)}$ is strictly controlled by the 2 -fold product of $M_{(\eta)}$.

Using the boundedness of $M_{(30 \rho), s}$ and $\mathcal{M}_{(5 \rho)}$, it is easy to see that Theorem 1.4 can be deduced from Lemma 2.1 and the following theorem.

Theorem 2.2. Consider $\omega \in \operatorname{Dini}(1)$, and let $T$ be a bilinear Calderón-Zygmund operator of type $\omega(t)$. The maximal bilinear operator $T^{\star}$ is defined as in (1.10). Then, for all $s>0, \rho \in[1, \infty)$, there exists a constant $C$ such that

$$
T^{\star}\left(f_{1}, f_{2}\right)(x) \leq C\left\{M_{(30 \rho), s}\left[T\left(f_{1}, f_{2}\right)\right](x)+\mathcal{M}_{(5 \rho)}\left(f_{1}, f_{2}\right)(x)\right\} .
$$

Proof of Theorem 2.2. Let $x \in \mathcal{X}$ be a point such that $\left|T\left(f_{1}, f_{2}\right)(x)\right|<\infty$ and $B_{x}$ be the biggest $\left(30 \rho, \beta_{30 \rho}\right)$-doubling ball centered at $x$ of the form $(30 \rho)^{-k} \varepsilon$, $k \geq 1$. Without loss of generality, we assume that $B_{x}:=B\left(x,(30 \rho)^{-k_{0}} \varepsilon\right), k_{0} \geq 1$ is a fixed number. Split $f_{i}=f_{i}^{0}+f_{i}^{\infty}$, where $f_{i}^{0}=f \chi_{6 B_{x}}$ and $f_{i}^{\infty}=f \chi \chi_{\mathcal{X} \backslash 6 B_{x}}$ $(i=1,2)$.

We claim that for any $z \in B_{x}$,

$$
\left|T_{\varepsilon}\left(f_{1}, f_{2}\right)(x)\right| \lesssim \mathcal{M}_{(5 \rho)}\left(f_{1}, f_{2}\right)(x)+\left|T\left(f_{1}, f_{2}\right)(z)-T\left(f_{1}^{0}, f_{2}^{0}\right)(z)\right| .
$$

We postpone the proof for (2.2) at the end of this section. First, let us describe how we can complete the proof for Theorem 2.2 by virtue of (2.2). It is obvious that

$$
\left|T_{\varepsilon}\left(f_{1}, f_{2}\right)(x)\right| \lesssim \mathcal{M}_{(5 \rho)}\left(f_{1}, f_{2}\right)(x)+\left|T\left(f_{1}, f_{2}\right)(z)\right|+\left|T\left(f_{1}^{0}, f_{2}^{0}\right)(z)\right|,
$$

for all $z \in B_{x}$. Fix now $0<s<\frac{1}{2}$, taking the $L^{s}\left(B_{x}, \frac{d \mu(x)}{\mu\left(B_{x}\right)}\right)$ - norm with respect to $z$, we have

$$
\begin{aligned}
\left|T_{\varepsilon}\left(f_{1}, f_{2}\right)(x)\right| \lesssim & \mathcal{M}_{(5 \rho)}\left(f_{1}, f_{2}\right)(x)+\left(\frac{1}{\mu\left(B_{x}\right)} \int_{B_{x}}\left|T\left(f_{1}, f_{2}\right)(z)\right|^{s} d \mu(z)\right)^{\frac{1}{s}} \\
& +\left(\frac{1}{\mu\left(B_{x}\right)} \int_{B_{x}}\left|T\left(f_{1}^{0}, f_{2}^{0}\right)(z)\right|^{s} d \mu(z)\right)^{\frac{1}{s}} .
\end{aligned}
$$

The assumption of boundedness in (1.9) leads to that

$$
\begin{aligned}
& \int_{B_{x}}\left|T\left(f_{1}^{0}, f_{2}^{0}\right)(z)\right|^{s} d \mu(z) \\
& =2 s \int_{0}^{\infty} \lambda^{2 s-1}\left|\left\{z \in B_{x}:\left|T\left(f_{1}^{0}, f_{2}^{0}\right)(z)\right|^{\frac{1}{2}}>\lambda\right\}\right| d \lambda \\
& \lesssim 2 s \int_{0}^{\infty} \lambda^{2 s-1} \min \left(\mu\left(B_{x}\right), \frac{W^{\frac{1}{2}}}{\lambda}\left(\prod_{i=1}^{2}\left\|f_{i} \chi_{6 B_{x}}\right\|_{L^{1}}\right)^{\frac{1}{2}}\right) d \lambda .
\end{aligned}
$$


Letting $R=W^{\frac{1}{2}}\left(\prod_{i=1}^{2}\left\|f_{i} \chi_{6 B_{x}}\right\|_{L^{1}}\right)^{\frac{1}{2}}$. We have

$$
\begin{aligned}
\int_{B_{x}}\left|T\left(f_{1}^{0}, f_{2}^{0}\right)(z)\right|^{s} d \mu(z) & \lesssim 2 s \int_{0}^{R / \mu\left(B_{x}\right)} \lambda^{2 s-1} \mu\left(B_{x}\right) d \lambda+2 s \int_{R / \mu\left(B_{x}\right)}^{\infty} \lambda^{2 s-2} R d \lambda \\
& \lesssim C_{s} R^{2 s} \mu\left(B_{x}\right)^{1-2 s} .
\end{aligned}
$$

Since $B_{x}$ is $\left(30 \rho, \beta_{30 \rho}\right)$-doubling ball, it follows that

$$
\begin{aligned}
& \left(\frac{1}{\mu\left(B_{x}\right)} \int_{B_{x}}\left|T\left(f_{1}^{0}, f_{2}^{0}\right)(z)\right|^{s} d \mu(z)\right)^{1 / s} \lesssim C_{s} W \frac{1}{\left(\mu\left(B_{x}\right)\right)^{2}} \prod_{i=1}^{2}\left\|f_{i} \chi_{6 B_{x}}\right\|_{L^{1}} \\
& \lesssim C_{s} W\left[\frac{\mu\left(5 \rho \times 6 B_{x}\right)}{\mu\left(B_{x}\right)} \frac{1}{\mu\left(5 \rho \times 6 B_{x}\right)}\right]^{2} \int_{6 B_{x}}\left|f_{1}\left(y_{1}\right)\right| d \mu\left(y_{1}\right) \int_{6 B_{x}}\left|f_{2}\left(y_{2}\right)\right| d \mu\left(y_{2}\right) \\
& \lesssim \mathcal{M}_{(5 \rho)}\left(f_{1}, f_{2}\right)(x) .
\end{aligned}
$$

Furthermore,

$$
\begin{aligned}
& \left(\frac{1}{\mu\left(B_{x}\right)} \int_{B_{x}}\left|T\left(f_{1}, f_{2}\right)(z)\right|^{s} d \mu(z)\right)^{1 / s} \\
& =\left(\frac{\mu\left(30 \rho B_{x}\right)}{\mu\left(B_{x}\right)} \frac{1}{\mu\left(30 \rho B_{x}\right)} \int_{B_{x}}\left|T\left(f_{1}, f_{2}\right)(z)\right|^{s} d \mu(z)\right)^{1 / s} \\
& \lesssim M_{(30 \rho), s}\left[T\left(f_{1}, f_{2}\right)\right](x) .
\end{aligned}
$$

According to the estimates of (2.3), (2.4), (2.5), we have

$$
T^{\star}\left(f_{1}, f_{2}\right)(x) \lesssim M_{(30 \rho), s}\left[T\left(f_{1}, f_{2}\right)\right](x)+\mathcal{M}_{(5 \rho)}\left(f_{1}, f_{2}\right)(x) .
$$

Now we turn our attention to the proof of (2.2). Since

$$
\begin{aligned}
& T\left(f_{1}, f_{2}\right)(z)-T\left(f_{1}^{0}, f_{2}^{0}\right)(z) \\
& =\int_{\max \left\{d\left(x, y_{1}\right), d\left(x, y_{2}\right)\right\}>6 \times(30 \rho)^{-k_{0} \varepsilon}} K\left(z, y_{1}, y_{2}\right) f_{1}\left(y_{1}\right) f_{2}\left(y_{2}\right) d \mu\left(y_{1}\right) d \mu\left(y_{2}\right),
\end{aligned}
$$

we only need to show that

$$
\begin{aligned}
& \left|T_{\varepsilon}\left(f_{1}, f_{2}\right)(x)-\int_{\max \left\{d\left(x, y_{1}\right), d\left(x, y_{2}\right)\right\}>6 \times(30 \rho)^{-k_{0} \varepsilon}} K\left(z, y_{1}, y_{2}\right) f_{1}\left(y_{1}\right) f_{2}\left(y_{2}\right) d \mu\left(y_{1}\right) d \mu\left(y_{2}\right)\right| \\
& \lesssim \mathcal{M}_{(5 \rho)}\left(f_{1}, f_{2}\right)(x) .
\end{aligned}
$$

Denoting $B(x, \varepsilon)$ by $B_{0}$, the corresponding estimate on the left of (2.6) is as below,

$$
\begin{aligned}
& \left|T_{\varepsilon}\left(f_{1}, f_{2}\right)(x)-\int_{\max \left\{d\left(x, y_{1}\right), d\left(x, y_{2}\right)\right\}>6 \times(30 \rho)^{-k_{0} \varepsilon}} K\left(z, y_{1}, y_{2}\right) f_{1}\left(y_{1}\right) f_{2}\left(y_{2}\right) d \mu\left(y_{1}\right) d \mu\left(y_{2}\right)\right| \\
& \lesssim\left|\int_{\max \left\{d\left(x, y_{1}\right), d\left(x, y_{2}\right)\right\}>\varepsilon}\left(K\left(x, y_{1}, y_{2}\right)-K\left(z, y_{1}, y_{2}\right)\right) f_{1}\left(y_{1}\right) f_{2}\left(y_{2}\right) d \mu\left(y_{1}\right) d \mu\left(y_{2}\right)\right|
\end{aligned}
$$




$$
\begin{aligned}
& +\mid \int_{\max \left\{d\left(x, y_{1}\right), d\left(x, y_{2}\right)\right\}>\varepsilon} K\left(z, y_{1}, y_{2}\right) f_{1}\left(y_{1}\right) f_{2}\left(y_{2}\right) d \mu\left(y_{1}\right) d \mu\left(y_{2}\right) \\
& -\int_{\max \left\{d\left(x, y_{1}\right), d\left(x, y_{2}\right)\right\}>6 \times(30 \rho)^{-k_{0} \varepsilon}} K\left(z, y_{1}, y_{2}\right) f_{1}\left(y_{1}\right) f_{2}\left(y_{2}\right) d \mu\left(y_{1}\right) d \mu\left(y_{2}\right) \mid \\
& :=\phi_{\mathbf{1}}+\phi_{\mathbf{2}} .
\end{aligned}
$$

We deal with the first term $\phi_{1}$, using the condition (1.5) and dividing the integral as follows,

$$
\begin{aligned}
\phi_{1} \lesssim & \int_{\substack{d\left(x, y_{1}\right)>\varepsilon \\
d\left(x, y_{2}\right)>\varepsilon}} \frac{f_{1}\left(y_{1}\right) f_{2}\left(y_{2}\right)}{[\lambda(x, d(x, \tilde{y}))]^{2}} \omega\left(\frac{d(x, z)}{\sum_{i=1}^{2} d\left(x, y_{i}\right)}\right) d \mu\left(y_{1}\right) d \mu\left(y_{2}\right) \\
& +\int_{\substack{d\left(x, y_{1}\right)>\varepsilon \\
d\left(x, y_{2}\right)<\varepsilon}}+\int_{\substack{d\left(x, y_{1}\right)<\varepsilon \\
d\left(x, y_{2}\right)>\varepsilon}} \frac{f_{1}\left(y_{1}\right) f_{2}\left(y_{2}\right)}{[\lambda(x, d(x, \tilde{y}))]^{2}} \omega\left(\frac{d(x, z)}{\sum_{i=1}^{2} d\left(x, y_{i}\right)}\right) d \mu\left(y_{1}\right) d \mu\left(y_{2}\right) \\
:= & I+I I+I I I .
\end{aligned}
$$

For term $I$, it will be that

$$
\begin{aligned}
I \lesssim & \sum_{k=1}^{\infty} \sum_{j=1}^{k-1} \int_{(30 \rho)^{j-1} \varepsilon<d\left(x, y_{2}\right) \leq(30 \rho)^{j} \varepsilon}\left|f_{2}\left(y_{2}\right)\right| \int_{(30 \rho)^{k-1} \varepsilon<d\left(x, y_{1}\right) \leq(30 \rho)^{k} \varepsilon} \frac{\left|f_{1}\left(y_{1}\right)\right|}{\left[\lambda\left(x, d\left(x, y_{1}\right)\right]^{2}\right.} \\
& \times \omega\left(\frac{d(x, z)}{d\left(x, y_{1}\right)}\right) d \mu\left(y_{1}\right) d \mu\left(y_{2}\right) \\
& +\sum_{k=1}^{\infty} \sum_{j=k}^{\infty} \int_{(30 \rho)^{j-1} \varepsilon<d\left(x, y_{2}\right) \leq(30 \rho)^{j} \varepsilon} \frac{\left|f_{2}\left(y_{2}\right)\right|}{\left[\lambda\left(x, d\left(x, y_{2}\right)\right)\right]^{2}} \omega\left(\frac{d(x, z)}{d\left(x, y_{2}\right)}\right) \\
& \times \int_{(30 \rho)^{k-1} \varepsilon<d\left(x, y_{1}\right) \leq(30 \rho)^{k} \varepsilon}\left|f_{1}\left(y_{1}\right)\right| d \mu\left(y_{1}\right) d \mu\left(y_{2}\right) \\
:= & I_{1}+I_{2} .
\end{aligned}
$$

Note that $z \in B_{x}=B\left(x,(30 \rho)^{-k_{0}} \varepsilon\right)$, we have $d(x, y)<(30 \rho)^{-k_{0}} \varepsilon$,

$$
\begin{aligned}
I_{1} \lesssim \sum_{k=1}^{\infty} \frac{1}{\left[\lambda\left(x,(30 \rho)^{k} \varepsilon\right)\right]^{2}} \int_{(30 \rho)^{k} B_{0}}\left|f_{1}\left(y_{1}\right)\right| d \mu\left(y_{1}\right) \omega\left(\frac{(30 \rho)^{-k_{0}} \varepsilon}{(30 \rho)^{k-1} \varepsilon}\right) \\
\quad \times \sum_{j=1}^{k-1} \int_{(30 \rho)^{j-1} \varepsilon<d\left(x, y_{2}\right) \leq(30 \rho)^{j} \varepsilon}\left|f_{2}\left(y_{2}\right)\right| d \mu\left(y_{2}\right) \\
\lesssim \sum_{k=1}^{\infty} \frac{1}{\left[\lambda\left(x,(30 \rho)^{k} \varepsilon\right)\right]^{2}} \int_{(30 \rho)^{k} B_{0}}\left|f_{1}\left(y_{1}\right)\right| d \mu\left(y_{1}\right) \omega\left(\frac{(30 \rho)^{-1} \varepsilon}{(30 \rho)^{k-1} \varepsilon}\right) \\
\quad \times \int_{(30 \rho)^{k-1} B_{0} \backslash B_{0}}\left|f_{2}\left(y_{2}\right)\right| d \mu\left(y_{2}\right) \\
\lesssim \sum_{k=1}^{\infty} \frac{\left[\mu\left(5 \rho(30 \rho)^{k} B_{0}\right)\right]^{2}}{\left[\lambda\left(x,(30 \rho)^{k} \varepsilon\right)\right]^{2}} \frac{1}{\left[\mu\left(5 \rho(30 \rho)^{k} B_{0}\right)\right]^{2}} \int_{(30 \rho)^{k} B_{0}}\left|f_{1}\left(y_{1}\right)\right| d \mu\left(y_{1}\right)
\end{aligned}
$$




$$
\begin{aligned}
& \times \int_{(30 \rho)^{k} B_{0}}\left|f_{2}\left(y_{2}\right)\right| d \mu\left(y_{2}\right) \omega\left((30 \rho)^{-k}\right) \\
\lesssim & \mathcal{M}_{(5 \rho)}\left(f_{1}, f_{2}\right)(x) \sum_{k=1}^{\infty} \omega\left((30 \rho)^{-k}\right) \\
\lesssim & \mathcal{M}_{(5 \rho)}\left(f_{1}, f_{2}\right)(x) .
\end{aligned}
$$

Here the series $\sum_{k=1}^{\infty} \omega\left((30 \rho)^{-k}\right)$ is equivalent to $\int_{0}^{1} \omega(t) \frac{d t}{t}$, where $\omega \in \operatorname{Dini}(1)$.

Next, we will deal with $I_{2}$.

$$
\begin{aligned}
I_{2} \lesssim \sum_{j=1}^{\infty} \int_{(30 \rho)^{j-1} \varepsilon<d\left(x, y_{2}\right) \leq(30 \rho)^{j} \varepsilon} \frac{\left|f_{2}\left(y_{2}\right)\right|}{\left[\lambda\left(x, d\left(x, y_{2}\right)\right)\right]^{2}} \omega\left(\frac{d(x, z)}{d\left(x, y_{2}\right)}\right) d \mu\left(y_{2}\right) \\
\quad \times \sum_{k=1}^{j} \int_{(30 \rho)^{k-1} \varepsilon<d\left(x, y_{1}\right) \leq(30 \rho)^{k} \varepsilon}\left|f_{1}\left(y_{1}\right)\right| d \mu\left(y_{1}\right) \\
\lesssim \sum_{j=1}^{\infty} \frac{\left[\mu\left(5 \rho(30 \rho)^{j} B_{0}\right)\right]^{2}}{\left[\lambda\left(x,(30 \rho)^{j} \varepsilon\right)\right]^{2}} \frac{1}{\left[\mu\left(5 \rho(30 \rho)^{j} B_{0}\right)\right]^{2}} \int_{(30 \rho)^{j} B_{0}}\left|f_{2}\left(y_{2}\right)\right| d \mu\left(y_{2}\right) \\
\quad \times \int_{(30 \rho)^{j} B_{0}}\left|f_{1}\left(y_{1}\right)\right| d \mu\left(y_{1}\right) \omega\left(\frac{(30 \rho)^{-1} \varepsilon}{(30 \rho)^{j-1} \varepsilon}\right) \\
\lesssim \mathcal{M}_{(5 \rho)}\left(f_{1}, f_{2}\right)(x) \sum_{j=1}^{\infty} \omega\left((30 \rho)^{-j}\right) \lesssim \mathcal{M}_{(5 \rho)}\left(f_{1}, f_{2}\right)(x) .
\end{aligned}
$$

The estimates of $I_{1}$ and $I_{2}$ lead to that $I \lesssim \mathcal{M}_{(5 \rho)}\left(f_{1}, f_{2}\right)(x)$ for $z \in B_{x}$.

In light of the symmetry of $I I$ and $I I I$, we only need to estimate $I I$.

$$
\begin{aligned}
I I \lesssim \sum_{k=1}^{\infty} \int_{(30 \rho)^{k-1} \varepsilon<d\left(x, y_{1}\right) \leq(30 \rho)^{k} \varepsilon} \frac{\left|f_{1}\left(y_{1}\right)\right|}{\left[\lambda\left(x, d\left(x, y_{1}\right)\right)\right]^{2}} \omega\left(\frac{d(x, z)}{d\left(x, y_{1}\right)}\right) d \mu\left(y_{1}\right) \\
\quad \times \int_{d\left(x, y_{2}\right)<\varepsilon}\left|f_{2}\left(y_{2}\right)\right| d \mu\left(y_{2}\right) \\
\quad \lesssim \sum_{k=1}^{\infty} \frac{1}{\left[\lambda\left(x,(30 \rho)^{k} \varepsilon\right)\right]^{2}} \int_{(30 \rho)^{k} B_{0}}\left|f_{1}\left(y_{1}\right)\right| d \mu\left(y_{1}\right) \omega\left(\frac{(30 \rho)^{-k_{0}} \varepsilon}{(30 \rho)^{k-1} \varepsilon}\right) \\
\quad \times \int_{B_{0}}\left|f_{2}\left(y_{2}\right)\right| d \mu\left(y_{2}\right) \\
\lesssim \mathcal{M}_{(5 \rho)}\left(f_{1}, f_{2}\right)(x) \sum_{k=1}^{\infty} \omega\left((30 \rho)^{-k}\right) \lesssim \mathcal{M}_{(5 \rho)}\left(f_{1}, f_{2}\right)(x) .
\end{aligned}
$$

According to the estimate of $I, I I$ and $I I I$, we get $\phi_{1} \lesssim \mathcal{M}_{(5 \rho)}\left(f_{1}, f_{2}\right)(x)$. 
It remains to deal with $\phi_{2}$. Noticing the range of integration, we have

$$
\begin{aligned}
\phi_{2} \lesssim & \int_{\substack{6 \times(30 \rho)^{-k_{0}} \varepsilon<d\left(x, y_{2}\right) \leq \varepsilon \\
6 \times(30 \rho)^{-k_{0}} \varepsilon<d\left(x, y_{1}\right) \leq \varepsilon}}\left|K\left(z, y_{1}, y_{2}\right) f_{1}\left(y_{1}\right) f_{2}\left(y_{2}\right)\right| d \mu\left(y_{1}\right) d \mu\left(y_{2}\right) \\
& +\int_{\substack{6 \times(30 \rho)^{-k_{0}} \varepsilon<d\left(x, y_{2}\right) \leq \varepsilon \\
d\left(x, y_{1}\right) \leq 6 \times(30 \rho)^{-k_{0}} \varepsilon}}\left|K\left(z, y_{1}, y_{2}\right) f_{1}\left(y_{1}\right) f_{2}\left(y_{2}\right)\right| d \mu\left(y_{1}\right) d \mu\left(y_{2}\right) \\
& +\int_{\begin{array}{c}
\left.d\left(x, y_{2}\right) \leq 6 \times(30 \rho)^{-k_{0}}\right) \\
6 \times(30 \rho)^{-k_{0}} \varepsilon<d\left(x, y_{1}\right) \leq \varepsilon
\end{array}}\left|K\left(z, y_{1}, y_{2}\right) f_{1}\left(y_{1}\right) f_{2}\left(y_{2}\right)\right| d \mu\left(y_{1}\right) d \mu\left(y_{2}\right) \\
:= & I V_{1}+I V_{2}+I V_{3} .
\end{aligned}
$$

Denote $6^{-\frac{1}{k_{0}}} \times 30 \rho$ by $\varrho_{k_{0}}$. For term $I V_{1}$, using the size condition (1.4), we have

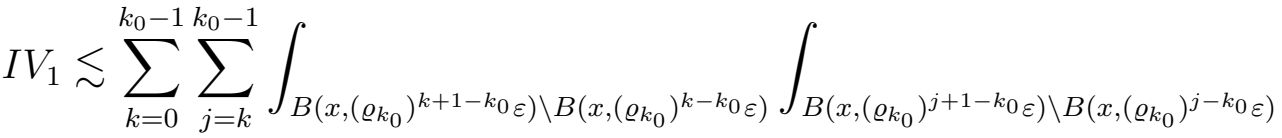

$$
\begin{aligned}
& \times \frac{1}{[\lambda(z, d(z, \tilde{y}))]^{2}}\left|f_{1}\left(y_{1}\right) f_{2}\left(y_{2}\right)\right| d \mu\left(y_{1}\right) d \mu\left(y_{2}\right) \\
& +\sum_{k=0}^{k_{0}-1} \sum_{j=0}^{k-1} \int_{B\left(x,\left(\varrho_{0}\right)^{k+1-k_{0}} \varepsilon\right) \backslash B\left(x,\left(\varrho_{k_{0}}\right)^{\left.k-k_{0} \varepsilon\right)}\right.} \int_{B\left(x,\left(\varrho_{k_{0}}\right)^{j+1-k_{0}} \varepsilon\right) \backslash B\left(x,\left(\varrho_{k_{0}}\right)^{\left.j-k_{0} \varepsilon\right)}\right.} \\
& \times \frac{1}{[\lambda(z, d(z, \tilde{y}))]^{2}}\left|f_{1}\left(y_{1}\right) f_{2}\left(y_{2}\right)\right| d \mu\left(y_{1}\right) d \mu\left(y_{2}\right) \\
& :=I V_{11}+I V_{12} \text {. }
\end{aligned}
$$

For $z \in B_{x}$, using the property (4) of $\lambda$ ( see Definition 1.2), we have

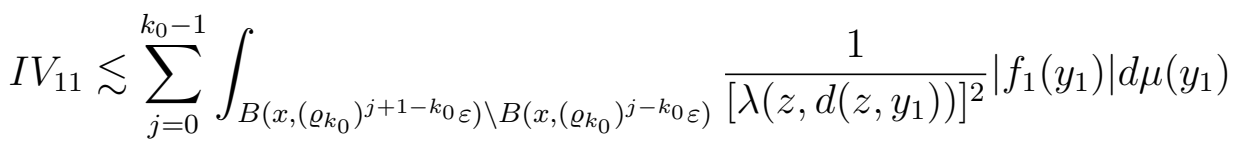

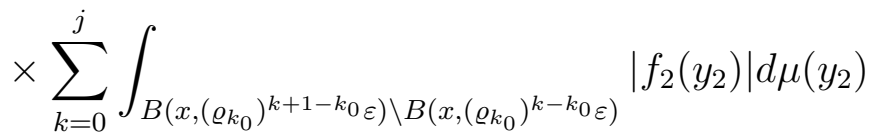

$$
\begin{aligned}
& \lesssim \sum_{j=0}^{k_{0}-1} \frac{\left[\mu\left(B\left(x, 5 \rho\left(\varrho_{k_{0}}\right)^{j+1-k_{0}} \varepsilon\right)\right)\right]^{2}}{\left[\lambda\left(x,\left(\varrho_{k_{0}}\right)^{j+1-k_{0} \varepsilon} \varepsilon\right]^{2}\right.} \frac{1}{\left[\mu\left(B\left(x, 5 \rho\left(\varrho_{k_{0}}\right)^{j+1-k_{0}} \varepsilon\right)\right)\right]^{2}} \\
& \times \int_{B\left(x,\left(\varrho_{0}\right)^{\left.j+1-k_{0 \varepsilon}\right)}\right.}\left|f_{1}\left(y_{1}\right)\right| d \mu\left(y_{1}\right) \int_{B\left(x,\left(\varrho_{k_{0}}\right)^{\left.j+1-k_{0 \varepsilon}\right)}\right.}\left|f_{2}\left(y_{2}\right)\right| d \mu\left(y_{2}\right) .
\end{aligned}
$$

By the similar methods used in [2], we get

$$
\sum_{j=0}^{k_{0}-1} \frac{\left[\mu\left(B\left(x, 5 \rho\left(\varrho_{k_{0}}\right)^{j+1-k_{0}} \varepsilon\right)\right)\right]^{2}}{\left[\lambda\left(x,\left(\varrho_{k_{0}}\right)^{j+1-k_{0}} \varepsilon\right)\right]^{2}} \lesssim C,
$$

thus,

$$
I V_{11} \lesssim \mathcal{M}_{(5 \rho)}\left(f_{1}, f_{2}\right)(x)
$$


An analogous argument with $I V_{11}$ lead to that

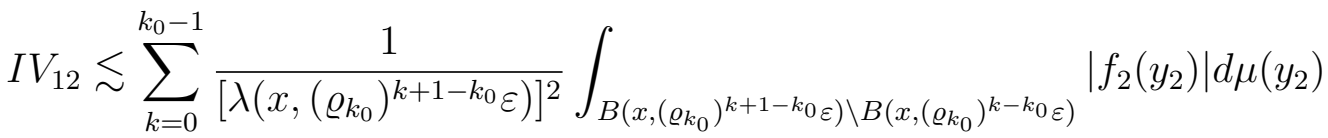

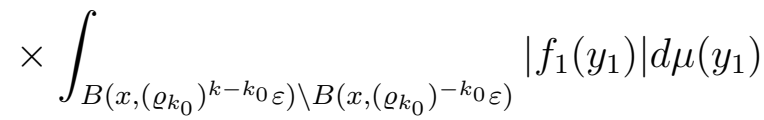

$$
\begin{aligned}
& \lesssim \mathcal{M}_{(5 \rho)}\left(f_{1}, f_{2}\right)(x) \text {. }
\end{aligned}
$$

Furthermore,

$$
I V_{1} \lesssim I V_{11}+I V_{12} \lesssim \mathcal{M}_{(5 \rho)}\left(f_{1}, f_{2}\right)(x)
$$

For term $I V_{2}$, considering the size condition (1.4) and it follows that

$$
\begin{aligned}
& I V_{2} \lesssim \int_{\substack{\left(\varrho_{k_{0}}\right)^{-k_{0}} \\
d\left(x, y_{2}\right)<\left(\varrho_{k_{0}}\right)^{-k_{0} \varepsilon}}} \frac{1}{[\lambda(z, d(z, \tilde{y}))]^{2}}\left|f_{1}\left(y_{1}\right) f_{2}\left(y_{2}\right)\right| d \mu\left(y_{1}\right) d \mu\left(y_{2}\right)
\end{aligned}
$$

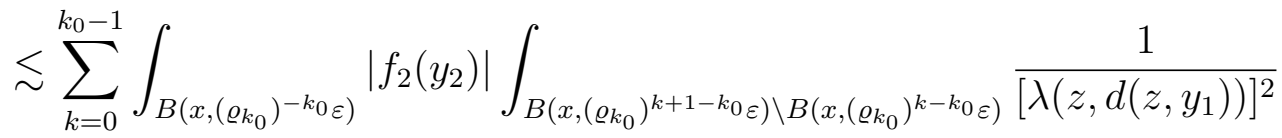

$$
\begin{aligned}
& \times\left|f_{1}\left(y_{1}\right)\right| d \mu\left(y_{1}\right) d \mu\left(y_{2}\right) \\
& \lesssim \sum_{k=0}^{k_{0}-1} \frac{\left[\mu\left(x, 5 \rho\left(\varrho_{k_{0}}\right)^{k+1-k_{0}} \varepsilon\right)\right]^{2}}{\left[\lambda\left(x,\left(\varrho_{k_{0}}\right)^{k+1-k_{0}} \varepsilon\right)\right]^{2}} \frac{1}{\left[\mu \left(x, 5 \rho\left(\varrho_{k_{0}}\right)^{\left.\left.k+1-k_{0} \varepsilon\right)\right]^{2}}\right.\right.} \int_{B\left(x,\left(\varrho_{k_{0}}\right)^{\left.k+1-k_{0} \varepsilon\right)}\right.}\left|f_{1}\left(y_{1}\right)\right| \\
& \times \int_{B\left(x,\left(\varrho_{k_{0}}\right)^{\left.k+1-k_{0} \varepsilon\right)}\right.}\left|f_{2}\left(y_{2}\right)\right| d \mu\left(y_{1}\right) d \mu\left(y_{2}\right) \\
& \lesssim \mathcal{M}_{(5 \rho)}\left(f_{1}, f_{2}\right)(x)
\end{aligned}
$$

Analogously, we have

$$
I V_{3} \lesssim \mathcal{M}_{(5 \rho)}\left(f_{1}, f_{2}\right)(x)
$$

The estimates of $I V_{1}, I V_{2}$ and $I V_{3}$ imply that $\phi_{2} \lesssim \mathcal{M}_{(5 \rho)}\left(f_{1}, f_{2}\right)(x)$. Collecting the estimates of $\phi_{1}$ and $\phi_{2}$, we get (2.6), then (2.2) holds obviously.

\section{Some Weighted estimates FOR $T^{\star}$}

3.1. Proof of Theorem 1.7. Firstly, we will introduce the weight class $A_{p}^{\rho}(\mu)$ and recall a lemma from [7]. In order to prove Theorem 1.7, we also need the following Theorem 3.3.

Definition 3.1. Let $\rho \in[1, \infty), p \in(1, \infty)$. A nonnegative $\mu$-measurable function $u$ is said to belong to $A_{p}^{\rho}(\mu)$ weight if there exists a positive constant $C$ such that for all balls $B \subset \mathcal{X}$,

$$
\left\{\frac{1}{\mu(\rho B)} \int_{B} u(x) d \mu(x)\right\}\left\{\frac{1}{\mu(\rho B)} \int_{B}[u(x)]^{1-p^{\prime}} d \mu(x)\right\}^{p-1} \lesssim C .
$$

A weight $u$ is called an $A_{1}^{\rho}(\mu)$ weight if there exists a positive constant $C$ such that for all balls $B \subset \mathcal{X}$,

$$
\frac{1}{\mu(\rho B)} \int_{B} u(x) d \mu(x) \leq C \inf _{y \in B} u(y)
$$


let $A_{\infty}^{\rho}(\mu)=\bigcup_{p=1}^{\infty} A_{p}^{\rho}(\mu)$.

Lemma 3.2. [7] Let $\rho \in[1, \infty), \mathcal{M}_{(\eta)}$ be defined as in (2.1), for any $p_{1}, p_{2} \in$ $[1, \infty)$ with $1 / p=1 / p_{1}+1 / p_{2}$ and $\vec{w}=\left(w_{1}, w_{2}\right) \in A_{\vec{p}}^{\rho}(\mu)$, the operator $\mathcal{M}_{(\eta)}$ is bounded from $L^{p_{1}}\left(w_{1}\right) \times L^{p_{2}}\left(w_{2}\right)$ into $L^{p, \infty}\left(v_{\vec{w}}\right)$.

Theorem 3.3. Consider $\omega \in \operatorname{Dini}(1)$. Suppose $K$ is a $\mu$-locally integrable function, which satisfies (1.4), (1.5), (1.6) and (1.7). Let $T^{\star}$ be defined as in (1.10). For any $\rho \in[1, \infty), u \in A_{2 p}^{\rho}(\mu)$ with $p \in[1 / 2, \infty)$, there exists a constant $C$ such that for all bounded functions $f_{1}, f_{2}$ with bounded support and $x \in \mathcal{X}$,

$$
\left\|T^{\star}\left(f_{1}, f_{2}\right)\right\|_{L^{p, \infty}(u)} \leq C\left\|\mathcal{M}_{(5 \rho)}\left(f_{1}, f_{2}\right)\right\|_{L^{p, \infty}(u)} .
$$

We postpone the proof for Theorem 3.3 at the end of this section.

Now we give the proof of Theorem 1.7. If $\vec{w} \in A_{\vec{p}}^{\rho}(\mu)$, noticing that $v_{\vec{w}}(x)=\prod_{i=1}^{2}\left[w_{i}(x)\right]^{p / p_{i}^{\prime}}$, using Hölder's inequality with $1=\frac{\left(p_{1}-1\right) p}{(2 p-1) p_{1}}+\frac{\left(p_{2}-1\right) p}{(2 p-1) p_{2}}$, we have

$$
\begin{aligned}
& \left\{\frac{1}{\mu(\rho B)} \int_{B} v_{\vec{w}}(x) d \mu(x)\right\}\left\{\frac{1}{\mu(\rho B)} \int_{B}\left[v_{\vec{w}}(x)\right]^{1-(2 p)^{\prime}} d \mu(x)\right\}^{2 p-1} \\
& \lesssim\left\{\frac{1}{\mu(\rho B)} \int_{B} v_{\vec{w}}(x) d \mu(x)\right\}\left\{\int_{B}\left[w_{1}^{-1}(x)\right]^{\frac{\left((2 p)^{\prime}-1\right) p}{p_{1}} \frac{(2 p-1) p_{1}}{\left(p_{1}-1\right) p}} d \mu(x)\right\}^{\frac{\left(p_{1}-1\right) p}{p_{1}}} \\
& \quad \times\left\{\int_{B}\left[w_{2}^{-1}(x)\right]^{\frac{\left((2 p)^{\prime}-1\right) p}{p_{2}} \frac{(2 p-1) p_{2}}{\left(p_{2}-1\right) p}} d \mu(x)\right\}^{\frac{\left(p_{2}-1\right) p}{p_{2}}}\left(\frac{1}{\mu(\rho B)}\right)^{2 p-1} \\
& =\left\{\frac{1}{\mu(\rho B)} \int_{B} v_{\vec{w}}(x) d \mu(x)\right\} \prod_{i=1}^{2}\left\{\frac{1}{\mu(\rho B)} \int_{B}\left[w_{i}(x)\right]^{1-p_{i}^{\prime}} d \mu(x)\right\}^{p / p_{i}^{\prime}} \\
& \lesssim C,
\end{aligned}
$$

which means that $v_{\vec{w}} \in A_{2 p}^{\rho}(\mu)$. Taking $u=v_{\vec{w}}$ in Theorem 3.3 and using Lemma 3.2 , it is easy to see that

$$
\begin{aligned}
\left\|T^{\star}\left(f_{1}, f_{2}\right)\right\|_{L^{p, \infty}\left(v_{\vec{w}}\right)} & \lesssim\left\|\mathcal{M}_{(5 \rho)}\left(f_{1}, f_{2}\right)\right\|_{L^{p, \infty}\left(v_{\vec{w}}\right)} \\
& \lesssim\left\|f_{1}\right\|_{L^{p_{1}}\left(w_{1}\right)}\left\|f_{2}\right\|_{L^{p_{2}}\left(w_{2}\right)},
\end{aligned}
$$

that is, Theorem 1.7 has been proved.

Before the proof of Theorem 3.3, we will introduce some maximal operators and give more lemmas. Denote by $m_{B} f$ the mean value of $f$ on $B$, namely, $m_{B} f=\frac{1}{\mu(B)} \int_{B} f(x) d \mu$. Let $s \in(0,1), \rho \in[1, \infty)$. For any fixed ball $B$ and $\mu$-measurable function $f$, define $M_{0, s ; B}^{\rho}$ by setting

$$
M_{0, s ; B}^{\rho}(f)=\inf \{t>0: \mu(\{y \in B:|f(y)>t|\})<s \mu(30 \rho B)\},
$$

when $\mu(B)>0$, and setting $M_{0, s ; B}^{\rho}(f)=0$ when $\mu(B)=0$.

For any $\mu$-measurable function $f$, the John-strömberg maximal operator $M_{0, s}^{\rho}$ is defined by setting, for all $x \in \mathcal{X}$,

$$
M_{0, s}^{\rho} f(x)=\sup _{B \text { is }\left(30 \rho, \beta_{30 \rho}\right)-\text { doubling }} M_{0, s ; B}^{\rho}(f),
$$


and the John-strömberg sharp maximal operator $M_{0, s}^{\rho, \sharp}$ is defined by

$$
M_{0, s}^{\rho, \sharp} f(x)=\sup _{B \ni x} M_{0, s ; B}^{\rho}\left[f-m_{\widetilde{B}}(f)\right]+\sup _{B, S \text { are }\left(30 \rho, \beta_{30 \rho}\right)-\text { doubling }} \frac{\left|m_{B}(f)-m_{S}(f)\right|}{1+K_{B, S}} .
$$

Let $r \in(0, \infty), \rho \in[1, \infty)$. Define the operator $M_{r}^{\rho, \sharp}$ by setting, for all $f \in$ $L_{l o c}^{r}(\mu)$ and $x \in \mathcal{X}$,

$$
\begin{aligned}
M_{r}^{\rho, \sharp} f(x)= & \sup _{B \ni x}\left[\frac{1}{\mu(30 \rho B)} \int_{B}\left|f(y)-m_{\widetilde{B}}(f)\right|^{r} d \mu(y)\right]^{\frac{1}{r}} \\
& +\sup _{B, S \text { are }\left(30 \rho, \beta_{30 \rho}\right)-\text { doubling }} \quad \frac{\left|m_{B}(f)-m_{S}(f)\right|}{1+K_{B, S}} .
\end{aligned}
$$

It is easy to show that for all $f \in L_{l o c}^{r}(\mu)$ and $x \in \mathcal{X}$,

$$
M_{0, s}^{\rho, \sharp} f(x) \leq s^{-1 / r} M_{r}^{\rho, \sharp} f(x) .
$$

These operators can be referred in [8]. In order to prove Theorem 3.3, we also need the following lemmas about the above maximal operators.

Lemma 3.4. [7] Let $\rho \in[1, \infty), s \in\left(0, \beta_{30 \rho}^{-1} / 4\right)$ and $B$ be a $\left(30 \rho, \beta_{30 \rho}\right)$-doubling ball. Then for all $\mu$-measurable functions $f$,

$$
\left|m_{B}(f)\right| \leq M_{0, s ; B}^{\rho}(f) .
$$

Lemma 3.5. [7] Let $\rho, p \in[1, \infty), s \in\left(0, \beta_{30 \rho}^{-1}\right)$. Then for all $\mu$-measurable function $f$ and $t \in(0, \infty)$,

(i) $\quad\{x \in \mathcal{X}:|f(x)|>t\} \subset\left\{x \in \mathcal{X}: M_{0, s}^{\rho}(f)(x) \geq t\right\} \cup E$ with $\mu(E)=0$.

(ii) For $u \in A_{p}^{\rho}(\mu)$, there exists a positive constant $C$, independent of $f$ and $t$, such that

$$
u\left(\left\{x \in \mathcal{X}: M_{0, s}^{\rho}(f)(x)>t\right\}\right) \leq C s^{-p} u(\{x \in \mathcal{X}:|f(x)|>t\}) .
$$

Lemma 3.6. [7] Let $\rho \in[1, \infty), s_{1} \in\left(0, \beta_{30 \rho}^{-1} / 4\right), 0<p<\infty, u \in A_{\infty}^{\rho}(\mu)$. There exists a constant $C_{1} \in(0,1)$, depending on $s_{1}$ and $u$, and a positive constant $C$ such that for any $s_{2} \in\left(0, C_{1} s_{1}\right)$,

(i) if $\mu(\mathcal{X})=\infty, f \in L^{p_{0}, \infty}(\mu)$ for some $p_{0} \in(0, \infty)$ and, for all $R \in(0, \infty)$,

$$
\sup _{t \in(0, R)} t^{p} u\left(\left\{x \in \mathcal{X}:\left|T^{\star}\left(f_{1}, f_{2}\right)(x)\right|>t\right\}\right)<\infty
$$

then

$$
\left\|M_{0, s_{1}}^{\rho}(f)\right\|_{L^{p, \infty}(u)} \leq C\left\|M_{0, s_{2}}^{\rho, \sharp}(f)\right\|_{L^{p, \infty}(u)} .
$$

(ii) If $\mu(\mathcal{X})<\infty, f \in L^{p_{0}, \infty}(\mu)$ with $p_{0} \in(0, \infty)$, then

$$
\left\|M_{0, s_{1}}^{\rho}(f)\right\|_{L^{p, \infty}(u)} \leq C\left\|M_{0, s_{2}}^{\rho, \sharp}(f)\right\|_{L^{p, \infty}(u)}+C u(\mathcal{X})\left[s_{1} \mu(\mathcal{X})\right]^{-p / p_{0}}\|f\|_{L^{p_{0}, \infty}(\mu)}^{p} .
$$

Next, we should establish a pointwise estimate for the operator $M_{r}^{\rho, \sharp}$, combining the above lemmas, we will deduce the main result in Theorem 3.3. 
Theorem 3.7. Let $\omega \in \operatorname{Dini}(1), K\left(x, y_{1}, y_{2}\right)$ be a locally integrable function defined away from the diagonal $x=y_{1}=y_{2}$ in $(\mathcal{X})^{3}$ and satisfies (1.4), (1.5), (1.6) and (1.7). Let $T^{\star}$ be defined as in (1.10), for any $\rho \in[1, \infty)$, there exists a constant $C$ such that for all bounded functions $f_{1}, f_{2}$ with bounded support and $x \in \mathcal{X}$,

$$
M_{r}^{\rho, \sharp}\left[T^{\star}\left(f_{1}, f_{2}\right)\right](x) \lesssim \mathcal{M}_{(5 \rho)}\left(f_{1}, f_{2}\right)(x) .
$$

In order to prove (3.2), as in the proof of Theorem 9.1 in [17], it suffices to prove that

$$
\left(\frac{1}{\mu(30 \rho B)} \int_{B}\left|T^{\star}\left(f_{1}, f_{2}\right)(y)-h_{B}\right|^{\delta} d \mu(y)\right)^{\frac{1}{\delta}} \lesssim \mathcal{M}_{(5 \rho)}\left(f_{1}, f_{2}\right)(x)
$$

and

$$
\left|h_{B}-h_{S}\right| \lesssim K_{B, S} \mathcal{M}_{(5 \rho)}\left(f_{1}, f_{2}\right)(x)
$$

hold for any balls $B \subset S$ with $x \in B:=B(x, r)$, where $S$ is a $\left(30 \rho, \beta_{30 \rho}\right)$-doubling ball, $0<\delta<1$. $h_{B}, h_{S}$ will be chosen later. At the end of this subsection, we will explain how to deduce (3.2) from (3.3) and (3.4).

Split each $f_{i}$ as $f_{i}=f_{i}^{0}+f_{i}^{\infty}, f_{i}^{0}=f_{i} \chi_{6 B}$ and $f_{i}^{\infty}=f_{i}-f_{i}^{0}, i=1,2$. It follows that

$$
\begin{aligned}
\left|T^{\star}\left(f_{1}, f_{2}\right)(y)\right| \lesssim & \left|T^{\star}\left(f_{1}^{0}, f_{2}^{0}\right)(y)\right|+\left|T^{\star}\left(f_{1}^{0}, f_{2}^{\infty}\right)(y)\right| \\
& +\left|T^{\star}\left(f_{1}^{\infty}, f_{2}^{0}\right)(y)\right|+\left|T^{\star}\left(f_{1}^{\infty}, f_{2}^{\infty}\right)(y)\right| .
\end{aligned}
$$

Now we set

$$
\begin{aligned}
& h_{B}=m_{B}\left(T^{\star}\left(f_{1}^{0}, f_{2}^{\infty}\right)+T^{\star}\left(f_{1}^{\infty}, f_{2}^{0}\right)+T^{\star}\left(f_{1}^{\infty}, f_{2}^{\infty}\right)\right), \\
& h_{S}=m_{S}\left(T^{\star}\left(f_{1}^{0}, f_{2}^{\infty}\right)+T^{\star}\left(f_{1}^{\infty}, f_{2}^{0}\right)+T^{\star}\left(f_{1}^{\infty}, f_{2}^{\infty}\right)\right) .
\end{aligned}
$$

In the following, we prove (3.3) first.

$$
\begin{aligned}
& \frac{1}{\mu(30 \rho B)} \int_{B}\left|T^{\star}\left(f_{1}, f_{2}\right)(y)-h_{B}\right|^{\delta} d \mu(y) \\
& \lesssim \frac{1}{\mu(30 \rho B)} \int_{B}\left|T^{\star}\left(f_{1}^{0}, f_{2}^{0}\right)(y)\right|^{\delta} d \mu(y) \\
& \quad+\frac{1}{\mu(30 \rho B)} \frac{1}{\mu(B)} \int_{B} \int_{B} \mid \sup _{\varepsilon>0}\left[T_{\varepsilon}\left(f_{1}^{\infty}, f_{2}^{0}\right)(y)-T_{\varepsilon}\left(f_{1}^{\infty}, f_{2}^{0}\right)(z)\right] \\
& \quad+\left.\sup _{\varepsilon>0}\left[T_{\varepsilon}\left(f_{1}^{0}, f_{2}^{\infty}\right)(y)-T_{\varepsilon}\left(f_{1}^{0}, f_{2}^{\infty}\right)(z)\right]\right|^{\delta} d \mu(z) d \mu(y) \\
& \quad+\frac{1}{\mu(30 \rho B)} \frac{1}{\mu(B)} \int_{B} \int_{B} \sup _{\varepsilon>0}\left|T_{\varepsilon}\left(f_{1}^{\infty}, f_{2}^{\infty}\right)(y)-T_{\varepsilon}\left(f_{1}^{\infty}, f_{2}^{\infty}\right)(z)\right|^{\delta} d \mu(z) d \mu(y) \\
& :=D+E+F .
\end{aligned}
$$

For the first term $D$, the estimate only involves the size condition (1.4). Kolmogorov's inequality and the assumption of end-point boundedness (1.9) tell us 
that

$$
\begin{aligned}
D^{\frac{1}{\delta}} & \lesssim\left(\frac{\mu(B)}{\mu(30 \rho B)}\right)^{\frac{1}{\delta}}\left\|T\left(f_{1} \chi_{6 B}, f_{2} \chi_{6 B}\right)\right\|_{L^{1 / 2, \infty}}\left(B, \frac{d \mu}{\mu(B)}\right) \\
& \lesssim\left(\frac{\mu(B)}{\mu(30 \rho B)}\right)^{\frac{1}{\delta}}\left[\frac{\mu(5 \rho \times 6 B)}{\mu(B)} \frac{1}{\mu(5 \rho \times 6 B)}\right]^{2} \prod_{i=1}^{2} \int_{6 B}\left|f_{i}\left(y_{i}\right)\right| d \mu\left(y_{i}\right) \\
& \lesssim\left(\frac{\mu(B)}{\mu(30 \rho B)}\right)^{\frac{1}{\delta}-2} \mathcal{M}_{(5 \rho)}\left(f_{1}, f_{2}\right)(x) \lesssim \mathcal{M}_{(5 \rho)}\left(f_{1}, f_{2}\right)(x) .
\end{aligned}
$$

Both $E$ and $F$ involve the regularity condition (1.5), we only give the estimate of $E$, in the same way, we can get the similar result of $F$. We consider the following two cases.

Case 1: $0<\varepsilon<5 r_{B}$. For any $y_{1} \in \mathcal{X} \backslash 6 B, y_{2} \in 6 B$ and any $t \in B(x, r)$, it is easy to see that $\max \left\{d\left(t, y_{1}\right), d\left(t, y_{2}\right)\right\}=d\left(t, y_{1}\right)>\varepsilon$. So using condition (1.5) for any $y, z \in B(x, r)$, we have

$$
\begin{aligned}
& \left|T_{\varepsilon}\left(f_{1}^{\infty}, f_{2}^{0}\right)(y)-T_{\varepsilon}\left(f_{1}^{\infty}, f_{2}^{0}\right)(z)\right| \\
& \lesssim \int_{6 B} \int_{\mathcal{X} \backslash 6 B}\left|K\left(y, y_{1}, y_{2}\right)-K\left(z, y_{1}, y_{2}\right)\right|\left|f_{1}\left(y_{1}\right) f_{2}\left(y_{2}\right)\right| d \mu\left(y_{1}\right) d \mu\left(y_{2}\right) \\
& \lesssim \int_{6 B} \int_{\mathcal{X} \backslash 6 B} \frac{1}{[\lambda(y, d(y, \tilde{y}))]^{2}} \omega\left(\frac{d(z, y)}{\sum_{i=1}^{2} d\left(y, y_{i}\right)}\right)\left|f_{1}\left(y_{1}\right)\right|\left|f_{2}\left(y_{2}\right)\right| d \mu\left(y_{1}\right) d \mu\left(y_{2}\right) \\
& \lesssim \int_{6 B} \frac{\left|f_{2}\left(y_{2}\right)\right|}{\lambda\left(y, d\left(y, y_{2}\right)\right)} d \mu\left(y_{2}\right) \sum_{k=1}^{\infty} \int_{6^{k+1} B \backslash 6^{k} B} \frac{\left|f_{1}\left(y_{1}\right)\right|}{\lambda\left(y, d\left(y, y_{1}\right)\right)} \omega\left(\frac{d(z, y)}{d\left(y, y_{1}\right)}\right) d \mu\left(y_{1}\right) \\
& \lesssim \frac{\mu(5 \rho \times 6 B)}{\lambda\left(x_{B}, 6 r_{B}\right)} \frac{1}{\mu(5 \rho \times 6 B)} \int_{6 B}\left|f_{2}\left(y_{2}\right)\right| d \mu\left(y_{2}\right) \\
& \quad \times \sum_{k=1}^{\infty} \omega\left(6^{-k}\right) \frac{\mu\left(5 \rho \times 6^{k+1} B\right)}{\lambda\left(x_{B}, 6^{k+1} r_{B}\right)} \frac{1}{\mu\left(5 \rho \times 6^{k+1} B\right)} \int_{6^{k+1} B}\left|f_{1}\left(y_{1}\right)\right| d \mu\left(y_{1}\right) \\
& \lesssim \mathcal{M}_{(5 \rho)}\left(f_{1}, f_{2}\right)(x) \sum_{k=1}^{\infty} \omega\left(6^{-k}\right) \lesssim \mathcal{M}_{(5 \rho)}\left(f_{1}, f_{2}\right)(x) .
\end{aligned}
$$

Case 2: $\varepsilon \geq 5 r_{B}$. For $y, z \in B(x, r), y_{1} \in \mathcal{X} \backslash 6 B, y_{2} \in 6 B$, we have the following estimates:

$$
\begin{aligned}
& \left|T_{\varepsilon}\left(f_{1}^{\infty}, f_{2}^{0}\right)(y)-T_{\varepsilon}\left(f_{1}^{\infty}, f_{2}^{0}\right)(z)\right| \\
& \lesssim \mid \int_{\max \left\{d\left(y, y_{1}\right), d\left(y, y_{2}\right)\right\}>\varepsilon} K\left(y, y_{1}, y_{2}\right) f_{1}^{\infty}\left(y_{1}\right) f_{2}^{0}\left(y_{2}\right) d \mu\left(y_{1}\right) d \mu\left(y_{2}\right) \\
& \quad-\int_{\max \left\{d\left(z, y_{1}\right), d\left(z, y_{2}\right)\right\}>\varepsilon} K\left(y, y_{1}, y_{2}\right) f_{1}^{\infty}\left(y_{1}\right) f_{2}^{0}\left(y_{2}\right) d \mu\left(y_{1}\right) d \mu\left(y_{2}\right) \mid \\
& \quad+\left|\int_{\max \left\{d\left(z, y_{1}\right), d\left(z, y_{2}\right)\right\}>\varepsilon}\left(K\left(y, y_{1}, y_{2}\right)-K\left(z, y_{1}, y_{2}\right)\right) f_{1}^{\infty}\left(y_{1}\right) f_{2}^{0}\left(y_{2}\right) d \mu\left(y_{1}\right) d \mu\left(y_{2}\right)\right| .
\end{aligned}
$$


On the basis of the estimates of $\phi_{1}$ and $\phi_{2}$ in section 2, it is easy to obtain

$$
\begin{aligned}
\left|T_{\varepsilon}\left(f_{1}^{\infty}, f_{2}^{0}\right)(y)-T_{\varepsilon}\left(f_{1}^{\infty}, f_{2}^{0}\right)(z)\right| \lesssim M_{(5 \rho)}\left(f_{1}, f_{2}\right)(x), \\
\left|T_{\varepsilon}\left(f_{1}^{0}, f_{2}^{\infty}\right)(y)-T_{\varepsilon}\left(f_{1}^{0}, f_{2}^{\infty}\right)(z)\right| \lesssim M_{(5 \rho)}\left(f_{1}, f_{2}\right)(x) .
\end{aligned}
$$

So we have $E \lesssim \mathcal{M}_{(5 \rho)}\left(f_{1}, f_{2}\right)(x)$.

As a consequence of the estimates of $D, E, F$, we can get (3.3).

Next, we should prove (3.4) for chosen $h_{B}$ and $h_{S}$. For any balls $B \subset S$ with $x \in B, S$ is a $\left(30 \rho, \beta_{30 \rho}\right)$ doubling ball. Noting that $S$ is a doubling ball, we have $S=\widetilde{S}$. Denote the smallest positive integer $N$ such that $6 S \subset(30 \rho)^{N} B$ by $N_{B, S}$. Let $f_{i}^{0}=f_{i} \chi_{6 B}, f_{i}^{N}=f_{i} \chi_{(30 \rho)^{N} B}, f_{i}^{B_{N}}=f_{i} \chi_{(30 \rho)^{N} B \backslash 6 B}, f_{i}^{\infty}=f_{i} \chi_{\mathcal{X} \backslash(30 \rho)^{N} B}$, $f_{i}^{S}=f_{i} \chi_{6 S}$ and $f_{i}^{S_{N}}=f_{i} \chi_{(30 \rho)^{N} B \backslash 6 S}$, write the difference $h_{B}-h_{S}$ in the following way:

$$
\begin{aligned}
\left|h_{B}-h_{S}\right| \leq & \left|m_{B}\left(T^{\star}\left(f_{1}^{B_{N}}, f_{2}^{0}\right)\right)\right|+\left|m_{B}\left(T^{\star}\left(f_{1}^{0}, f_{2}^{B_{N}}\right)\right)\right|+\left|m_{B}\left(T^{\star}\left(f_{1}^{B_{N}}, f_{2}^{B_{N}}\right)\right)\right| \\
& +\left|m_{B}\left(T^{\star}\left(f_{1}^{\infty}, f_{2}^{\infty}\right)\right)-m_{S}\left(T^{\star}\left(f_{1}^{\infty}, f_{2}^{\infty}\right)\right)\right| \\
& +\left|m_{B}\left(T^{\star}\left(f_{1}^{\infty}, f_{2}^{N}\right)\right)-m_{S}\left(T^{\star}\left(f_{1}^{\infty}, f_{2}^{N}\right)\right)\right| \\
& +\left|m_{B}\left(T^{\star}\left(f_{1}^{N}, f_{2}^{\infty}\right)\right)-m_{S}\left(T^{\star}\left(f_{1}^{N}, f_{2}^{\infty}\right)\right)\right| \\
& +\left|m_{S}\left(T^{\star}\left(f_{1}^{S}, f_{2}^{S_{N}}\right)\right)\right|+\left|m_{S}\left(T^{\star}\left(f_{1}^{S_{N}}, f_{2}^{S}\right)\right)\right|+\left|m_{S}\left(T^{\star}\left(f_{1}^{S_{N}}, f_{2}^{S_{N}}\right)\right)\right| \\
= & \sum_{i=1}^{9} G_{i} .
\end{aligned}
$$

Firstly, for term $G_{1}$, we deal with $T^{\star}\left(f_{1}^{B_{N}}, f_{2}^{0}\right)$, it follows from the size of kernel (1.4), for all $y \in B$,

$$
\begin{aligned}
\left|T^{\star}\left(f_{1}^{B_{N}}, f_{2}^{0}\right)(y)\right| & \lesssim \int_{(30 \rho)^{N} B \backslash 6 B} \int_{6 B} \frac{\left|f_{1}\left(y_{1}\right)\right|\left|f_{2}\left(y_{2}\right)\right|}{\left[\lambda(y, d(y, \tilde{y})]^{2}\right.} d \mu\left(y_{2}\right) d \mu\left(y_{1}\right) \\
\lesssim & {\left[\sum_{k=1}^{N_{B, S}-1} \int_{(30 \rho)^{k+1} B \backslash(30 \rho)^{k} B} \frac{\left|f_{1}\left(y_{1}\right)\right|}{\lambda\left(y, d\left(y, y_{1}\right)\right)} d \mu\left(y_{1}\right)+\int_{30 \rho B \backslash 6 B} \frac{\left|f_{1}\left(y_{1}\right)\right|}{\lambda\left(y, d\left(y, y_{1}\right)\right)} d \mu\left(y_{1}\right)\right] } \\
& \times \int_{6 B} \frac{\left|f_{2}\left(y_{2}\right)\right|}{\lambda\left(y, d\left(y, y_{2}\right)\right)} d \mu\left(y_{2}\right) \\
\lesssim & {\left[\sum_{k=1}^{N_{B, S}-1} \frac{1}{\lambda\left(5 \rho \times(30 \rho)^{k+1} B\right)} \frac{1}{\mu\left(5 \rho \times(30 \rho)^{k+1} B\right)} \int_{(30 \rho)^{k+1} B}\left|f_{1}\left(y_{1}\right)\right| d \mu\left(y_{1}\right)\right.}
\end{aligned}
$$




$$
\begin{aligned}
&\left.+\frac{\mu(5 \rho \times(30 \rho) B)}{\lambda\left(x_{B},(30 \rho) r_{B}\right)} \frac{1}{\mu(5 \rho \times(30 \rho) B)} \int_{(30 \rho) B}\left|f_{1}\left(y_{1}\right)\right| d \mu\left(y_{1}\right)\right] \\
& \times \frac{\mu(5 \rho \times 6 B)}{\lambda\left(x_{B}, 6 r_{B}\right)} \frac{1}{\mu(5 \rho \times 6 B)} \int_{6 B}\left|f_{2}\left(y_{2}\right)\right| d \mu\left(y_{2}\right) \\
& \lesssim\left(1+K_{B, S}\right) \mathcal{M}_{(5 \rho)}\left(f_{1}, f_{2}\right)(x) .
\end{aligned}
$$

Thus,

$$
G_{1} \lesssim\left(1+K_{B, S}\right) \mathcal{M}_{(5 \rho)}\left(f_{1}, f_{2}\right)(x) .
$$

Using the similar method, we get

$$
G_{2} \lesssim\left(1+K_{B, S}\right) \mathcal{M}_{(5 \rho)}\left(f_{1}, f_{2}\right)(x)
$$

Analogously,

$$
G_{7}+G_{8} \lesssim K_{B, S} \mathcal{M}_{(5 \rho)}\left(f_{1}, f_{2}\right)(x)
$$

Next, we consider $T^{\star}\left(f_{1}^{B_{N}}, f_{2}^{B_{N}}\right)$. For $y \in B$, according to the size condition (1.4), we have

$$
\begin{aligned}
& \left|T^{\star}\left(f_{1}^{B_{N}}, f_{2}^{B_{N}}\right)(y)\right| \\
& \lesssim \int_{(30 \rho)^{N} B \backslash 6 B} \int_{(30 \rho)^{N} B \backslash 6 B} \frac{\left|f_{1}\left(y_{1}\right)\right|\left|f_{2}\left(y_{2}\right)\right|}{[\lambda(y, d(y, \tilde{y}))]^{2}} d \mu\left(y_{1}\right) d \mu\left(y_{2}\right) \\
& \lesssim \int_{30 \rho B \backslash 6 B} \int_{30 \rho B \backslash 6 B} \frac{\left|f_{1}\left(y_{1}\right)\right|\left|f_{2}\left(y_{2}\right)\right|}{[\lambda(y, d(y, \tilde{y}))]^{2}} d \mu\left(y_{1}\right) d \mu\left(y_{2}\right) \\
& \quad+\sum_{k=1}^{N_{B, S}-1} \sum_{j=1}^{N_{B, S}-1} \int_{(30 \rho)^{k+1} B \backslash(30 \rho)^{k} B} \int_{(30 \rho)^{j+1} B \backslash(30 \rho)^{j} B} \frac{\left|f_{1}\left(y_{1}\right)\right|\left|f_{2}\left(y_{2}\right)\right|}{[\lambda(y, d(y, \tilde{y}))]^{2}} d \mu\left(y_{1}\right) d \mu\left(y_{2}\right) \\
& :=H_{1}+H_{2} .
\end{aligned}
$$

Noticing that $y_{1}, y_{2} \in 30 \rho B \backslash 6 B, y \in B$, it is obvious that $d\left(y, y_{i}\right) \geq 5 r_{B}(i=$ $1,2)$. The properties (4) of $\lambda$ implies that

$$
\begin{aligned}
H_{1} & \lesssim\left[\frac{\mu((5 \rho \times 30 \rho) B)}{\lambda\left(x_{B}, 5 r_{B}\right)}\right]^{2} \prod_{i=1}^{2} \frac{1}{\mu((5 \rho \times 30 \rho) B)} \int_{30 \rho B}\left|f_{i}\left(y_{i}\right)\right| d \mu\left(y_{i}\right) \\
& \lesssim \mathcal{M}_{(5 \rho)}\left(f_{1}, f_{2}\right)(x) .
\end{aligned}
$$

Taking advantage of the similar methods that used in the estimates of $I V_{1}$ in (2.8), we get

$$
H_{2} \leq \mathcal{M}_{(5 \rho)}\left(f_{1}, f_{2}\right)(x) .
$$

The estimates of $H_{1}$ and $H_{2}$ give us that

$$
G_{3} \lesssim \mathcal{M}_{(5 \rho)}\left(f_{1}, f_{2}\right)(x)
$$

Analogously,

$$
G_{9} \lesssim K_{B, S} \mathcal{M}_{(5 \rho)}\left(f_{1}, f_{2}\right)(x)
$$

For $G_{4}, G_{5}, G_{6}$, involving the kernel condition (1.5), similar argument as that of $F$ and $E$ in $(3.5)$ yields

$$
G_{4}+G_{5}+G_{6} \lesssim \mathcal{M}_{(5 \rho)}\left(f_{1}, f_{2}\right)(x) .
$$


Combining all the estimates for $G_{i}$ with $i \in\{1, \ldots, 9\}$, we get (3.4).

Finally, let us see how from (3.3) and (3.4) one gets (3.2). By the definition of $M_{r}^{\rho, \sharp}$, for $0<\delta<1$, we have

$$
\begin{aligned}
& \frac{1}{\mu(30 \rho B)} \int_{B}\left|T^{\star}\left(f_{1}, f_{2}\right)(y)-m_{\widetilde{B}}\left(T^{\star}\left(f_{1}, f_{2}\right)\right)\right|^{\delta} d \mu(y) \\
& \lesssim \frac{1}{\mu(30 \rho B)} \int_{B}\left|T^{\star}\left(f_{1}, f_{2}\right)(y)-h_{B}\right|^{\delta} d \mu(y)+\left|h_{B}-h_{\widetilde{B}}\right|^{\delta}+\left|m_{\widetilde{B}}\left(T^{\star}\left(f_{1}, f_{2}\right)\right)-h_{\widetilde{B}}\right|^{\delta} .
\end{aligned}
$$

Noting that $m_{B}(h)-c=m_{B}(h-c)$, so $\left|m_{\widetilde{B}}\left(T^{\star}\left(f_{1}, f_{2}\right)\right)-h_{\widetilde{B}}\right| \lesssim m_{\widetilde{B}}\left[T^{\star}\left(f_{1}, f_{2}\right)-\right.$ $\left.h_{\widetilde{B}}\right]$. Lemma 3.4 and (3.1) tell us that

$$
\begin{aligned}
\left|m_{\widetilde{B}}\left(T^{\star}\left(f_{1}, f_{2}\right)\right)-h_{\widetilde{B}}\right|^{\delta} & \lesssim\left|M_{0, s ; \widetilde{B}}^{\rho}\left[T^{\star}\left(f_{1}, f_{2}\right)-h_{\widetilde{B}}\right]\right|^{\delta} \\
& \lesssim \frac{1}{\mu(\widetilde{B})} \int_{\widetilde{B}}\left|T^{\star}\left(f_{1}, f_{2}\right)(y)-h_{\widetilde{B}}\right|^{\delta} d \mu(y) .
\end{aligned}
$$

Furthermore, for any two $\left(30 \rho, \beta_{30 \rho}\right)$-doubling balls $B \subset S$,

$$
\begin{aligned}
& \left|m_{B}\left(T^{\star}\left(f_{1}, f_{2}\right)\right)-m_{S}\left(T^{\star}\left(f_{1}, f_{2}\right)\right)\right| \\
& \lesssim\left|m_{B}\left[T^{\star}\left(f_{1}, f_{2}\right)-h_{B}\right]\right|+\left|h_{B}-h_{S}\right|+\left|m_{S}\left[T^{\star}\left(f_{1}, f_{2}\right)-h_{S}\right]\right| \\
& \lesssim M_{0, s ; B}^{\rho}\left[T^{\star}\left(f_{1}, f_{2}\right)-h_{B}\right]+\left|h_{B}-h_{S}\right|+M_{0, s ; S}^{\rho}\left[T^{\star}\left(f_{1}, f_{2}\right)-h_{S}\right] \\
& \lesssim\left(\frac{1}{\mu(30 \rho B)} \int_{B}\left|T^{\star}\left(f_{1}, f_{2}\right)(y)-h_{B}\right|^{\delta} d \mu(y)\right)^{\frac{1}{\delta}}+\left|h_{B}-h_{S}\right| \\
& \quad+\left(\frac{1}{\mu(30 \rho S)} \int_{S}\left|T^{\star}\left(f_{1}, f_{2}\right)(y)-h_{S}\right|^{\delta} d \mu(y)\right)^{\frac{1}{\delta}} .
\end{aligned}
$$

Since (3.3) and (3.4) have been proved, (3.2) follows directly.

3.2. Proof of Theorem 3.3. We invoke the idea from [7, 8]. Considering the following two cases.

Case I: $\mu(\mathcal{X})=\infty$. We claim that for all $R \in(0, \infty)$,

$$
\sup _{t \in(0, R)} t^{p} u\left(\left\{x \in \mathcal{X}:\left|T^{\star}\left(f_{1}, f_{2}\right)(x)\right|>t\right\}\right)<\infty .
$$

The above estimate can be obtained by employing the similar method used in dealing with (3.9) of [7], we omit the details. Now we conclude the proof of Theorem 3.3 in this case. Using Lemma 3.5 (i), Lemma 3.6(i), (3.1) and (3.2), we have

$$
\begin{aligned}
\left\|T^{\star}\left(f_{1}, f_{2}\right)\right\|_{L^{p, \infty}(u)} & \lesssim\left\|M_{0, s_{1}}^{\rho}\left[T^{\star}\left(f_{1}, f_{2}\right)\right]\right\|_{L^{p, \infty}(u)} \\
& \lesssim\left\|M_{0, s_{2}}^{\rho, \sharp}\left[T^{\star}\left(f_{1}, f_{2}\right)\right]\right\|_{L^{p, \infty}(u)} \\
& \lesssim\left\|s_{2}^{-\frac{1}{\delta}} M_{\delta}^{\rho, \sharp}\left[T^{\star}\left(f_{1}, f_{2}\right)\right]\right\|_{L^{p, \infty}(u)} \\
& \lesssim\left\|\mathcal{M}_{(5 \rho)}\left(f_{1}, f_{2}\right)\right\|_{L^{p, \infty}(u)}
\end{aligned}
$$


Case II: $\mu(\mathcal{X})<\infty$. We choose $p_{0}=1 / 2$ in Lemma 3.6. Then by the assumption of the end-point boundedness of $T: L^{1}(\mu) \times L^{1}(\mu) \rightarrow L^{1 / 2, \infty}(\mu)$, we see that for $u \in A_{2 p}^{\rho}(\mu)$,

$$
\begin{aligned}
u(\mathcal{X})[\mu(\mathcal{X})]^{-2 p}\left\|T^{\star}\left(f_{1}, f_{2}\right)\right\|_{L^{1 / 2, \infty}(\mu)}^{p} & \lesssim u(\mathcal{X})[\mu(\mathcal{X})]^{-2 p} \prod_{i=1}^{2}\left\|f_{i}\right\|_{L^{1}(\mu)}^{p} \\
& =u(\mathcal{X})\left(\lim _{r_{B} \rightarrow \infty} \prod_{i=1}^{2} \frac{1}{\mu(5 \rho B)} \int_{B}\left|f_{i}(y)\right| d \mu(y)\right)^{p} \\
& \lesssim u(\mathcal{X})\left(\mathcal{M}_{(5 \rho)}\left(f_{1}, f_{2}\right)(x)\right)^{p} \\
& \lesssim \sup _{t>0} t^{p} u\left(\left\{x \in \mathcal{X}: \mathcal{M}_{(5 \rho)}\left(f_{1}, f_{2}\right)>t\right\}\right),
\end{aligned}
$$

The main result of Theorem 3.3 again follows from Lemma 3.5 (i), Lemma 3.6(ii), (3.1) and (3.2). This completes the proof of the theorem.

Acknowledgement. This work was supported by National Natural Science Foundation of China(Grant Nos. 11271330 and 11471288). The authors are very grateful to the referees for their valuable comments. The authors also want to express their deep thanks to professor Dashan Fan and Guoen Hu for their helpful suggestions.

\section{REFERENCES}

1. Á. Bényi, D. Maldonado, A.R. Nahmod and R.H. Torres, Bilinear paraproducts revisited, Math. Nachr. 283 (2010), no. 9, 1257-1276.

2. T.A. Bui and X.T. Duong, Hardy Spaces, regularized BMO Spaces and the boundedness of Calderón-Zygmund operators on non-homogeneous Spaces, J. Geom. Anal. 23 (2013), no. 2, 895-932.

3. J. Chen and D. Fan, Some bilinear estimates, J. Korean Math. Soc. 46 (2009), no. 3, 609-620.

4. J. Chen and G. Lu, Hömander type theorems for multi-linear and multi-parameter Fourier multiplier operators with limited smoothness, Nonlinear Anal. 101 (2014), 98-112.

5. L. Grafakos and R.H. Torres, Multilinear Calderón-Zygmund theory, Adv. Math. 165 (2002), no. 1, 124-164.

6. L. Grafakos and R.H. Torres, Maximal operator and weighted norm inequalities for multilinear singular integrals, Indiana Univ. Math. J. 51 (2002), no. 5, 1261-1276.

7. G. Hu, Y. Meng and D. Yang, Weighted norm inequalities for multilinear CalderónZygmund operators on non-homogeneous metric measure spaces, Forum Math. 26 (2014), no. 5, 1289-1322.

8. G. Hu and D. Yang, Weighted norm inequality for maximal singular integrals with nondoubling measure, Studia Math. 187 (2008), no. 2, 101-123.

9. T. Hytönen, A framework for non-homogeneous analysis on metric spaces, and the RBMO spaces of Tolsa, Publ. Mat. 54 (2010), no. 2, 485-504.

10. M. Lacey and J. Metcalfe, Paraproducts in one and several parameters, Forum Math. 19 (2007), no. 2, 325-351.

11. A.K. Lerner, S. Ombrosi, C. Pérez, R.H. Torres and R. Trujillo-Gonzalez, New maximal functions and multiple weights for the multilinear Calderón-Zygmund theory, Adv. Math. 220 (2009), no. 2, 1222-1264.

12. G. Lu and P. Zhang, Multilinear Calderón-Zygmund operators with kernels of Dini's type and applications, Nonlinear Anal. 107 (2014), 92-117. 
13. D. Maldonado and V. Naibo, Weighted norm inequalities for paraproducts and bilinear pseudodifferential operators with mild regularity, J. Fourier Anal. Appl. 15 (2009), no. 2, 218-261.

14. C. Muscalu, J. Pipher, T. Tao and C. Thiele, Bi-parameter paraproducts, Acta Math. 193 (2004), no. 2, 269-296.

15. J. Orobitg and C. Pérez, $A_{P}$ weights for nondoubling measures in $\mathbb{R}^{n}$ and applications, Trans. Amer. Math. Soc. 354 (2002), no. 5, 2013-2033.

16. X. Tao and T. Zheng, Multilinear commutators of fractional integrals over Morrey spaces with non-doubling measures, Nonlinear Differ. Equ. Appl. 18 (2011), no. 3, 287-308.

17. X. Tolsa, BMO, $H^{1}$ and Calderón-Zygmund operators for non doubling measures, Math. Ann. 319 (2001), no. 1, 89-149.

18. K. Yabuta, Generalization of Calderón-Zygmund operators, Studia Math. 82 (1985), no. 1, $17-31$.

19. T. Zheng, X. Tao and X. Wu, Bilinear Calderón-Zygmund operators of type $\omega(t)$ on non-homogeneous space, J. Inequal. Appl. 2014, 2014:113.

1 Department of Mathematics, Zhejiang University of Science and Technology, Hangzhou 310023, P. R. China.

E-mail address: zhengtao@zju.edu.cn

E-mail address: wangzheng10.17@163.com;

E-mail address: xwltc123@163.com

2 Department of Mathematics, Zhejiang University, Hangzhou 310027, P. R. China. 